\title{
Comparison of The Diagnostic Accuracy of Magnetic Resonance Imaging (MRI), Transabdominal Ultrasound (TAS), Transvaginal Ultrasound (TVS) In Characterizing The Uterine Mass Lesions.
}

\author{
Devimeenal Jagannathan ${ }^{1}$, Arun Dilip Subramanian ${ }^{2}$ \\ ${ }^{\text {I}}$ (Professorand Head,Department Of Radiodiagnosis, Government Kilpauk Medical College/ Dr.MGR.Medical \\ University,Tamilnadu,India) \\ ${ }_{2}^{2}$ (Senior Resident,,Department Of Radiodiagnosis, Government Kilpauk Medical College/ Dr.MGR.Medical \\ University, Tamilnadu, India)
}

\begin{abstract}
:
Introduction: Mass lesions are seen in the uterus in 15 to $20 \%$ of women in the reproductive age group. Transabdominal(TAS) ultrasound(USG) is the screening imaging modality of choice. Definitive characterisation of the site, location, endometrial distorsion and number of lesions are important to decide on the treatment

Aims and objectives: We compared the sensitivity and specificity of Magnetic resonance Imaging (MRI), TAS, Transvaginal ultrasound (TVS) in detecting and characterizing the uterine mass lesions.

Material and methods: We prospectively studied patients with suspected uterine masses.They were subjected to TAS, TVS and MRI examination. Depending upon the final diagnosis they underwent endometrial curettage, cervical biopsy, myomectomy or hysterectomy. Final histopathological report was taken as gold standard to compare the final diagnosis of each imaging modality.

Results: The total patients were subgrouped into 5 depending upon the histopathological diagnosis.

For detection of myometrial mass lesions, the diagonal agreement between the TVS and MRI is $96 \%$. In classifying the site of myometrial mass lesions, the diagonal agreement between TVS and MRI is 67\%. But all tiny submucosal lesions are seen in MRI.

For pickingup cystic degeneration within the fibroids TVS and MRI are useful and for calcification TAS or TVS more useful. In positive cases of adenomyosis minimal JZ thickness was $9 \mathrm{~mm}$. JZ myometrial ratio was above 23 $\%$. The sensitivity of detecting adenomyosis in TAS, TVS and MRI respectively is 33\%, 58\% and 92\%. In detecting endometrial Ca, MRI is 100\% sensitive \& 97\%. Specific. Prolapsing fundal fibroid with cyst in the pedicle and cervical polyp are diagnosed correctly with MRI.

Conclusion: USG is the best screening modality. Calcific degeneration is better identified by TAS and TVS

Pelvic MR Imaging compared to TAS and TVS is a well tolerated, non invasive and accurate imaging modality for characterising, localizing and detecting number \& extent of uterine mass lesions and for staging
\end{abstract}

Keywords : Adenomyosis, Cervical polyp, Endometrial ca, Fibroid, Uterine mass

\section{Introduction}

Mass lesions in the uterus are a commonly encountered problem all over the world. 15 to $20 \%$ of women in the reproductive age group have mass lesions in the uterus1. Transabdominal(TAS) ultrasound is the screening imaging modality of choice. Definitive characterisation of the lesion is important to decide on the treatment modality like myomectomy or hysterectomy in case of myomas. But in case of Adenomyosis, hysterectomy is the treatment of choice for definitive cure 1,2. This states the need for a definitive preoperative diagnosis. In cases of submucosal fibroid, if the endometrial surface circumference is more than $50 \%$ it can be resected hysteroscopically2. Otherwise it should be resected laparoscopically. The same way for intramural fibroids, depending on the number and site of the lesion, laparoscopic resection or hysterectomy can be planned. It states the need for exact location and extent of the lesion by various measurements like size and circumference. Even though it can be answered by Transvaginal ultrasound (TVS), it has the limitation of operator dependence and large lesions cannot be brought within the field of TVS (Fig 1).

Hysterosonosalphingography is useful only in endometrial lesions. CT is utilized to evaluate uterine lesion mainly in staging the Carcinoma(CA). The continuing need for better imaging modality for definitive characterisation of uterine mass lesions lead to the use of Magnetic Resonance Imaging (MRI) as a diagnostic tool in preoperative evaluations. 


\section{Materials And Methods}

A prospective study of 52 patients with suspected uterine masses referred from Obstetrics and Gynaecology department is done from January 2016 to August 2016. All patients were subjected to TAS, TVS and MRI examination. Depending upon the final diagnosis they underwent endometrial curettage, cervical biopsy, myomectomy or hysterectomy. Final histopathological report was taken as gold standard to compare the findings and the final diagnosis of each imaging modality. Grey scale TAS and TVS were performed using a Alpha 6 Aloka unit; equipped with 3.5 mega hertz convex probe and 5.0 mega hertz Transvaginal probe. MRI was performed using 1.5 tesla scanner Signa 1.5 HDXT,GE corporation, US) .A scout coronal section was obtained to plan for sagittal views. oblique coronal and oblique axial sections were planned using sagittal slices ( along the axis of uterus and perpendicular to it ).The sequences used were T1 weighted sagittal, (TR 700ms , TE 10ms, Slice thickness 4mm, FOV 300, Matrix size 256x256), T2 weighted sagittal, coronal and axial (TR 3250ms, TE $97 \mathrm{~ms}$, Slice thickness 4mm, FOV 300, Matrix size 256x256). Fat Saturation in cases of Endometriosis and Ovarian dermoid was used as optional Sequence.

All patients who were willing to undergo all three tests and willing for surgery were included in the study. Patients who did not undergo all three tests not operated or non-availability of the histopathological examination report, patient with MR incompatible devices or implants and patients with Claustrophobia were excluded. This study was approved by the ethics committee of our institute and an informed consent for all the scans were taken from all patients included in it. The data was analysed. Diagonal agreement, Cohen's Kappa, Z value are calculated comparing two modalities like TAS - MRI, TVS - MRI, TAS - TVS in detecting mass lesions in the uterus. Percentage of diagonal agreement implies number of cases detected as same final outcome regarding the presence of mass in both the modalities. Sensitivity, specificity, correctclassification, misclassification, Kappa values calculated for each modality for all cases coming under each final diagnosis and finally compared. Cohen's Kappa is used to compare the correlation between the modalities. The values of Kappa were classified as 0.0-0.2 no correlation, 0.2-0.4 fair correlation, 0.4-0.6 moderate correlation, 0.6-0.8 good correlation, $0.8-1.0$ very good correlation

\section{Results}

52 patients were included in the study age group ranging from 30 to $65 \mathrm{yrs} .68 \%$ of the patients were in the premenopausal age group, $33 \%$ of the patients were in the postmenopausal age group.Their respective age distributions were 32 patients in 30 to $40 \mathrm{yrs}, 8$ in 40 to $50 \mathrm{yrs}, 8$ in 50 to $60 \mathrm{yrs}$ and 4 over $60 \mathrm{yrs}$, with the mean age of $42.5 \mathrm{yrs}$ (Table1). Out of 52 patients in our study, 36 patients $(69.2 \%)$ presented with complaints of pain, $19(37 \%)$ patients had bleeding, 3 patients (6\%) had mass felt by themselves, 16 patients $(31 \%)$ had discharge PV, 1 patient (1.9\%) with loss of weight, 3 patients (5.8\%) with loss of appetite(Fig 2). Few had more than one complaint. But the common presentations were pain abdomen, bleeding PV and discharge PV.

The total patients were subgrouped into 5 depending upon the histopathological diagnosis (Fig 3). Adenomyosis -12 patients; Fibroid -24 patients; Endometrial carcinoma -3 patients, Carcinoma cervix 10patients, Cervical polyp -2 patients. Among the total patients 17 had adnexal lesions, 4 patients had mass lesions and 13 had cysts. Among 13 patients with cysts, one patient had endometriotic cyst. Sensitivity and specificity were calculated for each modality in each subgroup and compared. First in detection of myometrial mass lesions, 3 sets of data (Table 2) are derived by comparing two modalities in each. When comparing TAS and MRI in myometrial mass lesion detection the diagonal agreement was $71 \%$. It means only $71 \%$ of the mass lesions seen in MRI is seen in the USG. Diagonal agreement between the TVS and MRI is 96\%. Diagonal agreement between the TAS and TVS is 75\%.In classifying myometrial mass lesions(Table 3), the diagonal agreement between USG and MRI is 54\%, between TVS and MRI is 67\%. This is because small sub mucosal lesion detection is zero with TAS and only two submucosal lesions among 6 were diagnosed in TVS. But all are seen in MRI.Among fibroids 2, 4, 5 patients showed cystic degeneration with TAS,TVS and MRI respectively.9,10,4 patients show calcification in TAS,TVS and MRI respectively.

Among total 52 cases, the sensitivity of USG in detecting fibroid is $79 \%$, specificity is $86 \%$, and kappa is 0.65 (adequate), $\mathrm{P}<0.01$ stating that it is statistically significant.

TVS in detecting fibroid the sensitivity is $100 \%$, specificity is $89 \%$, and kappa is $0.89, \mathrm{P} \leq 0.01$. In MRI the sensitivity is $100 \%$, specificity is $96 \%$ (Fig 4).Among 12 patients who had adenomyosis histopathologically 10 of them had pain, 5 patients had bleeding PV, 5 patients had both, 4 had discharge PV. Dysmenorrhoea was present in 10 of them and 1 patient had infertility. All patients were in the age group of 31 39 years In the TAS 8 of them had inhomogenous myometrial echo. 4 patients had suspicious intramural anechoic lesion in the abdominal scan (Fig 5a ).They were taken as positive for adenomyosis by TAS.

7 patients had anterior, posterior wall myometrial asymmetry in both TAS and TVS. 11 of them had myometrial inhomogeneity in TVS. All 7 patients had posterior wall myometrial cyst(fig 5a \&b ). 3 of them had both anterior and posterior wall cyst in TVS. In MRI among 12 positive cases all had ill defined mass in the 
myometrium (Fig 5c). All patients had altered signal intensities in the myometrium. 3 of them had hyperintense signal in both $\mathrm{T}_{1}$ and $\mathrm{T}_{2}$ weighted sequences suggestive of haemorrhagic endometrial glands. In the rest 9, 4 of them had hyperintense signals in $\mathrm{T}_{2}$ weighted sequences and 5 patients had hypointense areas which were ill defined in $\mathrm{T}_{2}$ weighted sequences. All patients had junctional zone (JZ) above $10 \mathrm{~mm}$ with $\mathrm{JZ}$ myometrial ratio of above $23 \%$ in positive cases of adenomyosis. In one patient who had focal adenomyosis the JZ thickness was $9 \mathrm{~mm}$. Among 12 total patients of adenomyosis one patient had fibroid and adenomyosis, 9 had diffuse adenomyosis, 2 had focal adenomyoma(fig 6). In detecting endometrial Ca(Fig7a,b,c) in our study, MRI is $100 \%$ sensitive but specificity is $97 \%$. Kappa is 0.85 with very good agreement. $\mathrm{P} \leq 0.01$ statistically significantIn case of Ca cervix(Fig 8a,b,c) as all the patients came at or after stage IIb, all cases were detected correctly by all the modalities. one case of Ca cervix had large left iliac adenopathy which was eroding the iliac bone. Two cases were diagnosed as cervical polyp by MRI. One case was prolapsing fundal fibroid with cyst in the pedicle(Fig9a,b,c) correctly diagnosed by MRI. It was diagnosed as nabothian cyst in TVS. Another case was fibroid polyp arising just above the endo / ecto cervical junction, better delineated in coronal MRI(fig $10 \mathrm{a}, \mathrm{b}, \mathrm{c})$

\section{Discussion}

Most of the patients in our study population were in the reproductive age group. This is because benign mass lesions are more commonly seen in this age group ${ }^{1}$.In our study specificity of TVS in detecting fibroid is $89 \%$, because in one case reported in TVS as having both adenomyosis and fibroid turned out to be a focal adenomyoma with diffuse adenomyosis in MRI. No fibroid was found. In USG many intramural lesions of adenomyosis are reported as fibroid reducing the specificity.Duiak etal ${ }^{3}$ study on uterine leiomyomas in the infertile patient reported the sensitivity of MRI accuracy as $94 \%$ and concluded that MR imaging is superior to USG or HSG for preoperatively locating leiomyomas. Zawin etal ${ }^{4}$ observed accurate determination of uterine volume was possible in all cases by MRI, but was limited on USG in uterus larger than 140 cc. Marked enlargement obscured the visualization of contour abnormalities in USG. Endometrial stripe and JZ could not be adequately visualised in 21 out of 23 USG examinations. MRI detected more submucosal lesions and concluded that MRI is superior to the ultrasound in pelvic examination of women with leiomyoma. This correlates with our study in which TAS did not detect any submucosal lesions. Transvaginal detected two out of 6 lesions and MRI detected all 6 lesions. All 12 patients who had adenomyosis were in the age group of 31-39 years in our study, fits in with the VG Padubidri et $\mathrm{al}^{1}$ report that adenomyosis is usually seen in parous women around the age of 40 who present with menorrhagia and progressively increasing dysmenorrhoea. Pelvic discomfort and dyspareunia are the other symptoms of adenomyosis ${ }^{1}$.

Among 12 positive cases of adenomyosis comparing to histopthological examination, sensitivity of TAS is $33 \%$ in detecting adenomyosis. TVS is $58 \%$ and MRI is $92 \%$. This is because we have taken only positive cases of adenomyosis. No normal cases were sent for imaging. Specificity cannot be calculated when only positive cases were taken into account. But among three modalities MRI is more sensitive in detecting adenomyosis. Even then the sensitivity is only $92 \%$ because one case considered as fibroid by MRI turned out to be focal adenomyoma with normal $\mathrm{JZ}$ thickness. Kang $\mathrm{S}$ et $\mathrm{al}^{5}$ study on specificity of $5 \mathrm{~mm}$ as the maximum normal uterine $\mathrm{JZ}$ thickness in detecting adenomyosis concluded that if a diagnosis of adenomyosis is based solely on $\mathrm{JZ}$ thickness of $5 \mathrm{~mm}$ as upper limit of normal may result in a high false positive rate. Our study has showed $10 \mathrm{~mm}$ as minimal $\mathrm{JZ}$ thickness in positive cases of adenomyosis .

Similar to our results a study by Ascher SM et al $^{6}$ on 20 women, showed that $15 / 17$ cases were correctly diagnosed in MR. One false positive and 2 false negative diagnosis were made in MR. With TVS 9/17 cases were correctly diagnosed. One false positive and 8 false negative and reported most frequent causes of false negative diagnosis with TVs is misinterpretation of adenomyosis as leiomyoma.

In our study one case considered as fibroid by MRI turned out to be focal adenomyoma. Only 7out of 12 cases were diagnosed correctly as adenomyosis in TVS because of misinterpretation of adenomyosis as leiomyoma.This is emphasized by many studies Tamai $\mathrm{K} \mathrm{etal}^{7}$ studied the MR Imaging findings of adenomyosis and correlated with histopathologic features and diagnostic pitfalls. They reported myometrial contractions, leiomyoma, adenomatoid tumour, metastasis, endometrial carcinoma $(\mathrm{Ca})$ and endometrial stromal sarcoma were few pitfalls in diagnosis of adenomyosis. They concluded that familiarity with various appearances of adenomyosis and the possible pitfalls in differential diagnosis were more helpful in making confident diagnosis of adenomyosis.Mark AS etal ${ }^{8}$ study on 21 premenopausal patients, 8 had adenomyosis, 12 had fibroids and 1 was normal. All 8 adenomyosis were diagnosed on MRI. 10/12fibroid correctly diagnosed in MRI. In 2 cases

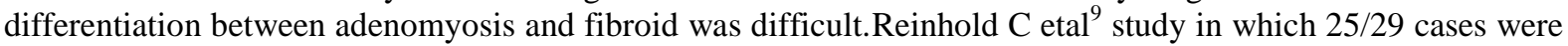
diagnosed positive for adenomyosis in TVS with a sensitivity of $86 \%$, specificity of $86 \%$ positive predictive value of $71 \%$ and negative predictive value of $94 \%$.In TAS $21 / 25(84 \%)$ had heterogenous echotexture, $3(12 \%)$ had hypoechoic areas with cysts, heterogenous areas in myometrium in $1(4 \%)$ patient 
In Byun JY etal ${ }^{10}$ study $66.7 \%$ (30cases) had diffuse adenomyosis and $33.3 \%$ (15) had focal adenomyoma. In diffuse adenomyosis $\mathrm{JZ}$ was $7-37 \mathrm{~mm}$. High signal intensity foci was seen in $\mathrm{T}_{2}$ weighted Images only in 4 and on both $\mathrm{T}_{1}$ and $\mathrm{T}_{2}$ weighted images in 11 of focal adenomyoma. In our study 10 had diffuse adenomyosis, 2 had focal adenomyosis. JZ thickness varied from 10 to $22 \mathrm{~mm}$.

One patient had associated chocolate cyst (Endometriosis) in our study. Diagnostic accuracy of endometriosis was improved with addition of fat saturated images together with conventional images ${ }^{11}$.

In detecting endometrial CA MRI is $100 \%$ sensitive in our study. The reduction in specificity is because one case considered as endometrial $\mathrm{Ca}$ stage 1a was normal by histopathology in our study. It showed small hyper intense foci breeching the JZ. On retrospective review of the image and correlating with the clinical history and HPE report, it was concluded that the MR finding may be due to the fractional curettage done two days earlier. No contrast imaging was done at that time. This suggests that MRI finding does not obviate the need of endometrial biopsy and fractional curettage which is important in deciding about the final diagnosis. Buyak E etal ${ }^{12}$ emphasized that fractional curettage was the best method for detecting endometrial abnormalities in women with post menopausal bleeding. Yamashita $\mathrm{Y}$ etal ${ }^{13}$ and associates prospectively studied assessment of myometrial invasion by endometrial CA in 40 patients by TVS versus contrast enhanced MR imaging and concluded that contrast enhanced $\mathrm{T}_{1}$ weighted $\mathrm{MR}$ imaging is significantly superior.

False positivity in $\mathrm{T}_{2}$ weighted imaging was due to distension of endometrial cavity by pyometra, the presence of myoma, atrophy of the myometrium and poor tumour- myometrium contrast ${ }^{13}$. Increased accuracy in contrast enhanced images was due to increased ability to distinguish between tumour, endometrial cavity, tumour invaded myometrium and residual myometrium ${ }^{13}$. If contrast enhanced MRI had been performed in our case false positive diagnosis of endometrial CA would have been avoided.

Ascher $\mathrm{SM}^{14}$ etal studied uterine changes after dilatation and curettage the in MR Imaging and reported that marked hypointense curvilinear areas in the endometrial canal on day 2 scans in all patients, $(\mathrm{P}=.0002)$. By day 7 either they decreased in size or completely resolved. No significant change in the width of the endometrial stripe or signal intensity of the $\mathrm{JZ}$ or myometrium after D\&C. The JZ was focally disrupted in one patient who had complication of uterine perforation. Yamashita Yetal ${ }^{15}$ studied dynamic contrast images,obtained every 30 seconds in the sagittal plane., The tumor-myometrial contrast was marked at 120 seconds in endometrial $\mathrm{CA}$ (contrast-to-noise ratio $[\mathrm{C} / \mathrm{N}], 26.0$ than that on postcontrast T1-weighted images $(\mathrm{C} / \mathrm{N}, 10.0)$ and on $\mathrm{T} 2$-weighted images $(\mathrm{C} / \mathrm{N}, 2.14)$. They found dynamic postcontrast MR images were superior in differentiating viable tumors from necrosis or residual secretion in the endometrial cavity. The accuracy of T2-weighted imaging and dynamic imaging was $67.9 \%$ and $84.9 \%$, respectively in predicting tumor and myometrial invasion ${ }^{15}$. Number of positive cases of endometrial CA is only 3in our study is a limitation. So there is a need to evaluate more positive cases.

MR plays a main role only in staging CA cervix, because if vaginal wall or parametrial involvement is detected it upgrades the staging. Parametrial invasion is detected mainly in oblique $\mathrm{T}_{2}$ axial or contrast enhanced $\mathrm{T}_{1}$ weighted axial images because breech in the hypointense line of the outer stroma is better seen in axial images ${ }^{16}$. Vaginal and body extensions are better seen in sagittal and coronal images.

Haematometrocolpos are also better seen in $T_{2}$ weighted or $T_{1}$ weighted images. Fat sat $T_{2}$ weighted Images and gradient images better demonstrated haematometrocolpos in two of our cases, haematosalphinx(Fig $8 \mathrm{c}$ ) is also better visualized in coronal and sagittal images. This finding can be made use of in diagnosing haematosalphinx in case of primary amenorrhoea. Outwater GK etal ${ }^{17}$ studied 41 surgically proven patients with dilated fallopian tubes for MR characteristics of dilated tubes in $\mathrm{T}_{1}, \mathrm{~T}_{2}$ weighted FSE sequences , thickened tubal wall, mucosal folds and signal intensity of intratubal fluid. Hyperintense tubal fluid in $\mathrm{T}_{1}$ weighted sequence was seen in endometriosis suggestive of haemorrhagic content.One case of CA cervix with large left iliac adenopathy was thought to be an adnexal mass by TAS. But the lesion was not reachable in TVS. Normal structures were seen between the uterus and the mass in transvaginal scan suggesting that the origin of the mass is not from adnexa. But MRI due to it's large FOV, was able to cover the entire area and proved it to be only iliac node with displacement of iliac vessels with thrombosis. This is the additional advantage of MRI in staging CA cervix and better detection of extensions.

Diagnosis of cervical polyp is a clinical one and can be suspected with TVS and TAS. Prolapsed submucosal leiomyoma into cervical or vaginal canal were better characterized and exact length of the pedicle can be measured and given to the surgeon in coronal MRI images ${ }^{18}$ (Fig 10c). 


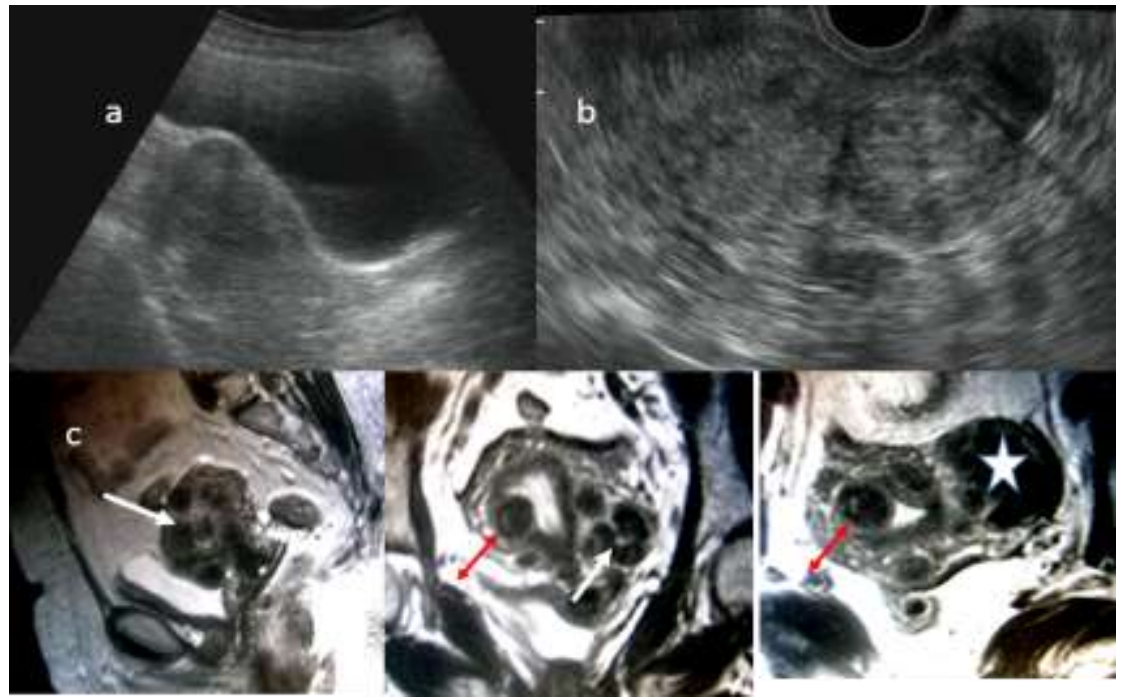

Fig 1a.TAS shows bulky uterus with mass lesion, 1b. TVS shows large subserous fibroid,1c. MRI saggital, coronal and axial views show multiple well defined hypointense fibroids in intra mural (Arrow), subserosal (star) and sub mucosal (double headed arrow) location.

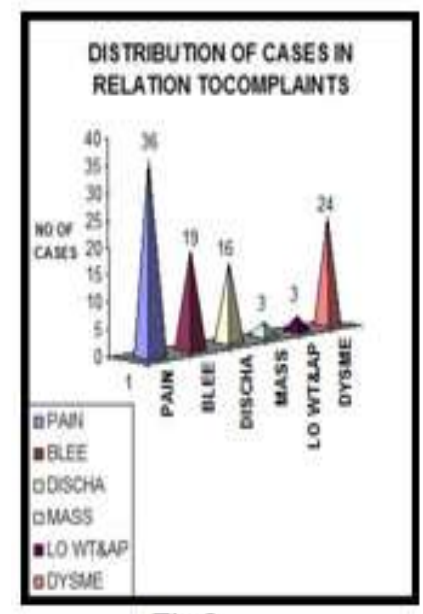

Fig 2

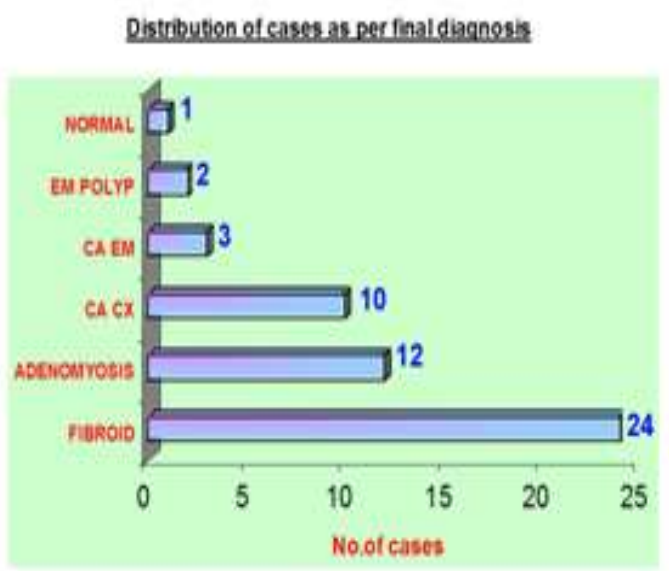

Fig 3 .

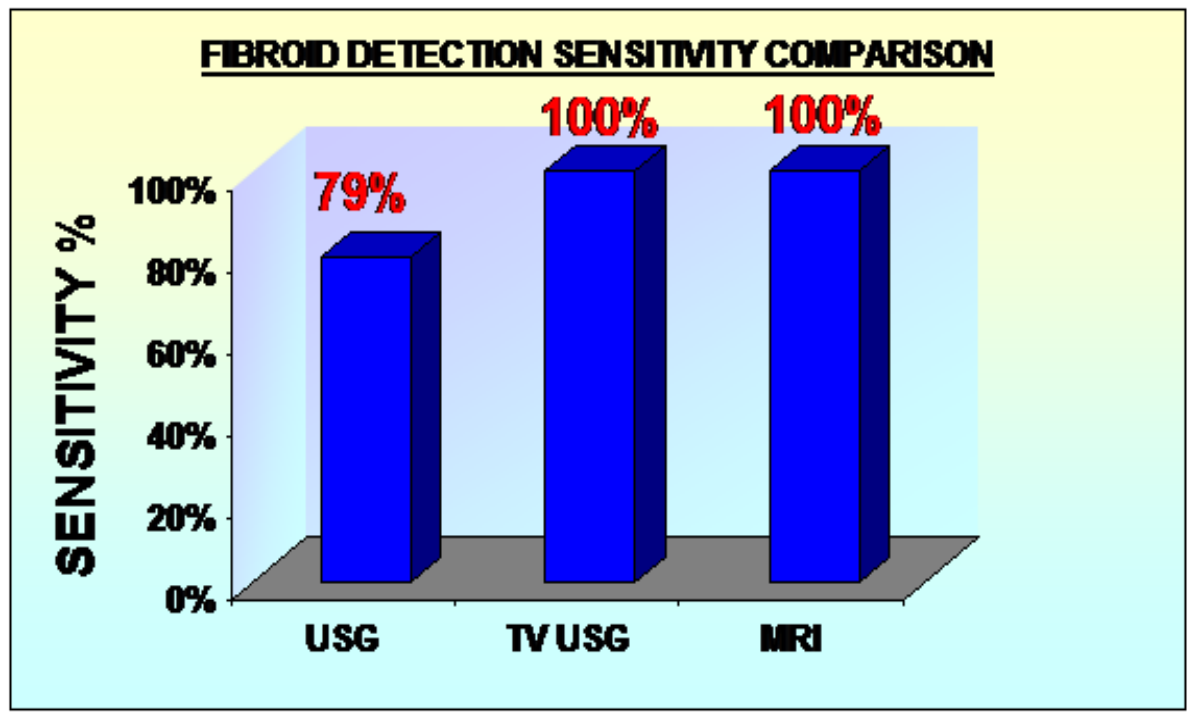

Fig 4.Comparison of sensitivity of all 3 modalities in detecting fibroid. 


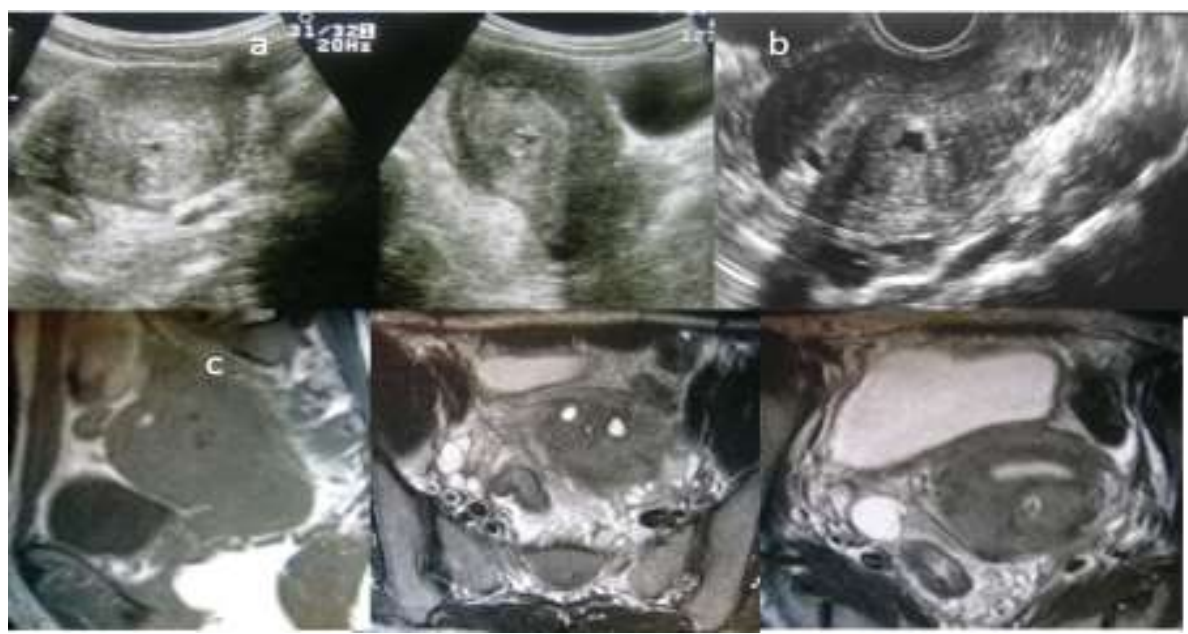

Fig 5 A case of adenomyosis. 5a.TAS shows bulky posterior myometrium with illdefined mass and cystic area, 5b. TVS shows subtle mass displacing the endometrial stripe anteriorly ,5c. MRI saggital T1, axial and coronal T2 images show ill defined hypointense mass with T1 and T2 hyperintense foci (Arrow) suggestive of hemorrhagic component and Junctional zone (double headed arrow) thickening
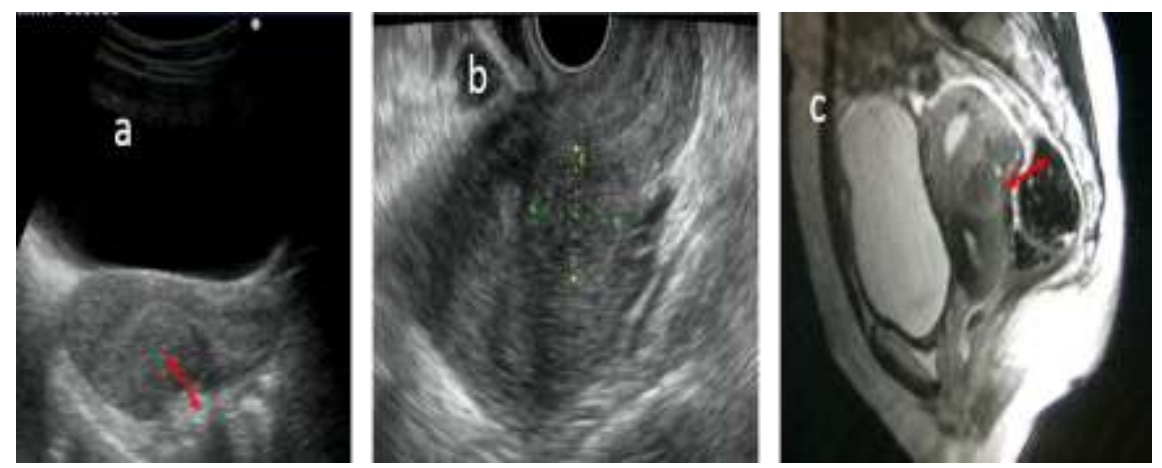

Fig 6a.TAS shows anteverted retroflexed uterus and suspicious posterior myometrial mass (double headed arrow). 6b. TVS shows hypoechoic mass displacing endometrial stripe anteriorly ,6c. MRI saggital T2 images show ill defined hypointense mass with hyperintense foci (double headed arrow) suggestive of focal adenomyoma.

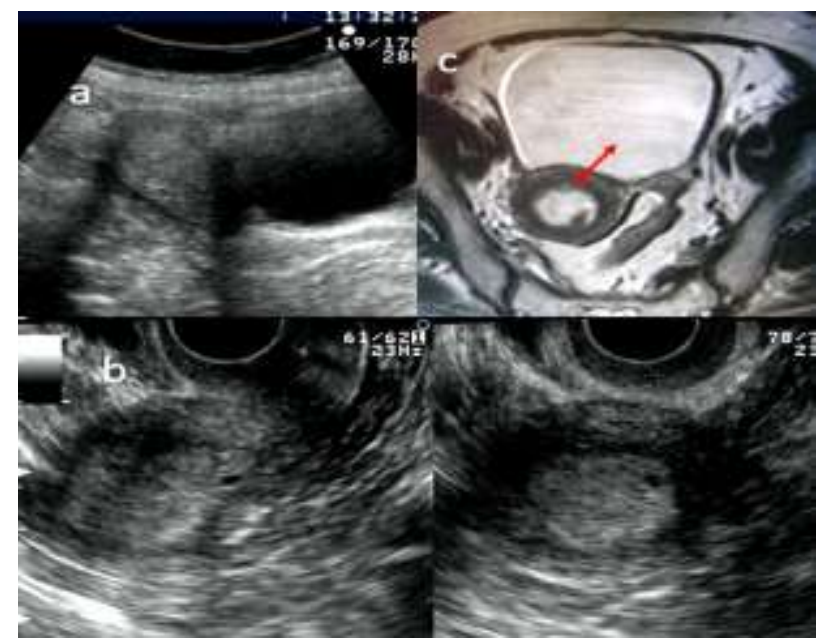

Fig 7a.TAS shows normal sized uterus with significant endometrial thickening. 7b. TVS saggital and axial image shows significant endometrial thickening and cystic area with junctional zone lobulation, 7c. MRI axial T2 images show hypointense fibrous core and significant endometrial thickening with breech in anterior junctional zone (double headed arrow) suggestive of endometrial polyp with carcinoma. 


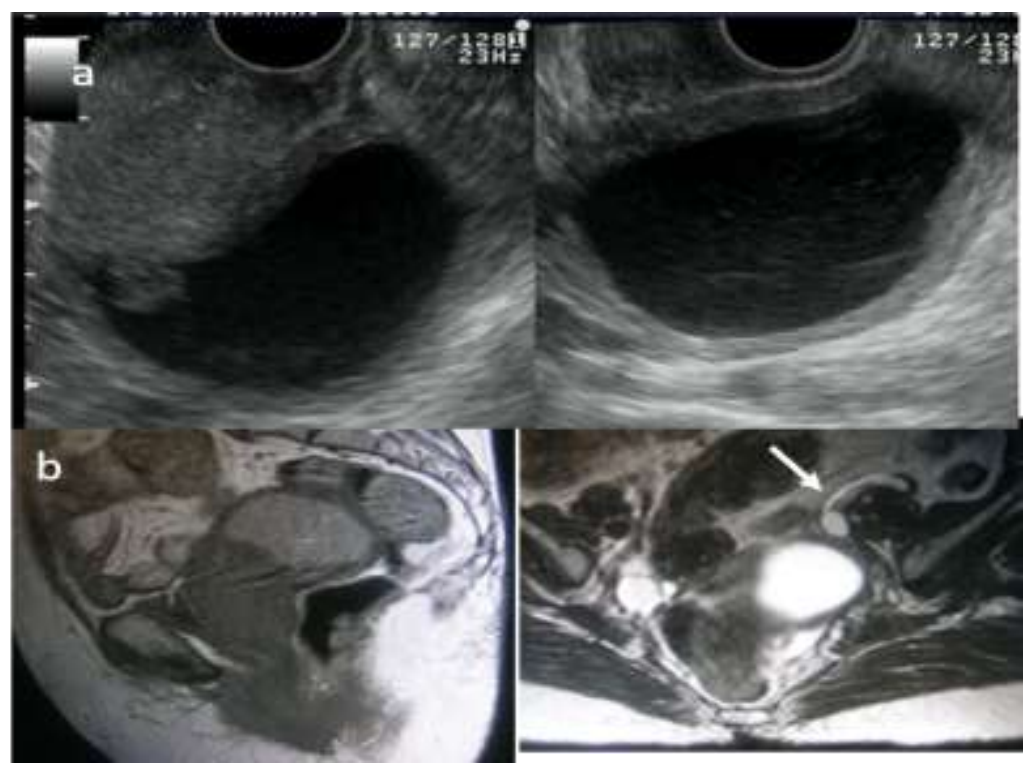

Fig 8a. TVS saggital and axial image shows significant endometrial fluid collection with mass in the cervix. , 8b. MRI Saggital T1 images show hyperintense endometrial fluid collection suggestive of haematometra with cervical mass and no pericervical stranding. 8c. MRI axial T2 images show haematometra with curvi linear hyperintense fallopian tube (haematosalphinx) (double headed arrow)
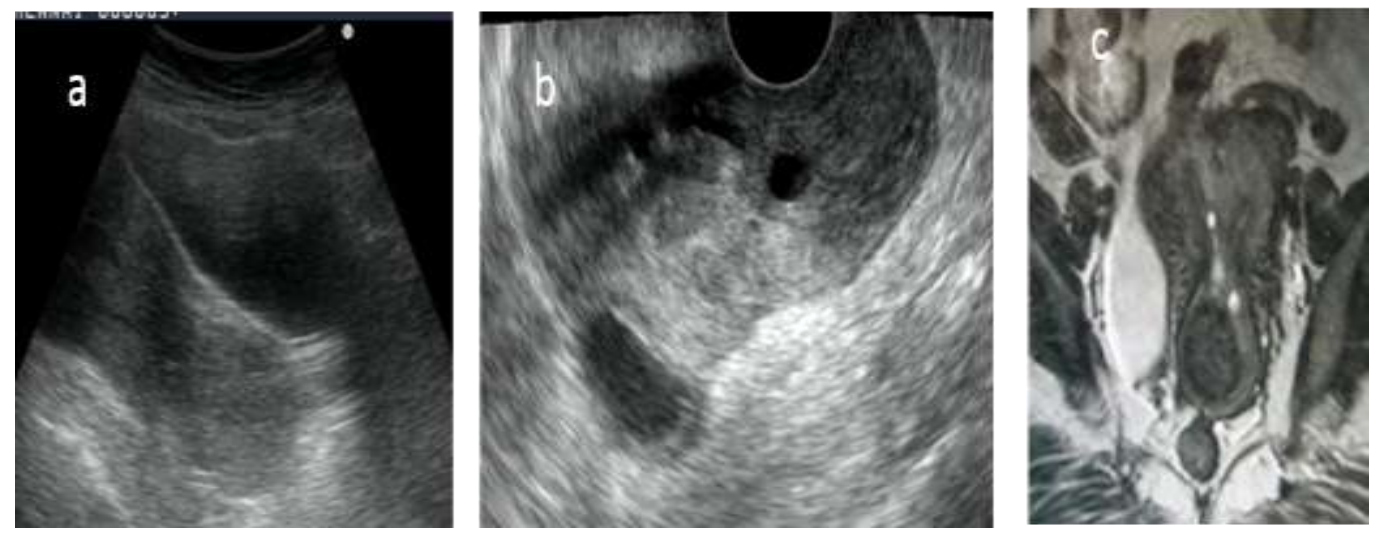

Fig 9a.TAS shows inadequate visualization of fundus of the uterus and bulky cervix. 9b. TVS saggital image shows absent fundal contour and bulky hypo echoic cervix with small cyst., 9c. MRI coronal T2 images show prolasing fundal fibroid with small cyst in the pedicle.
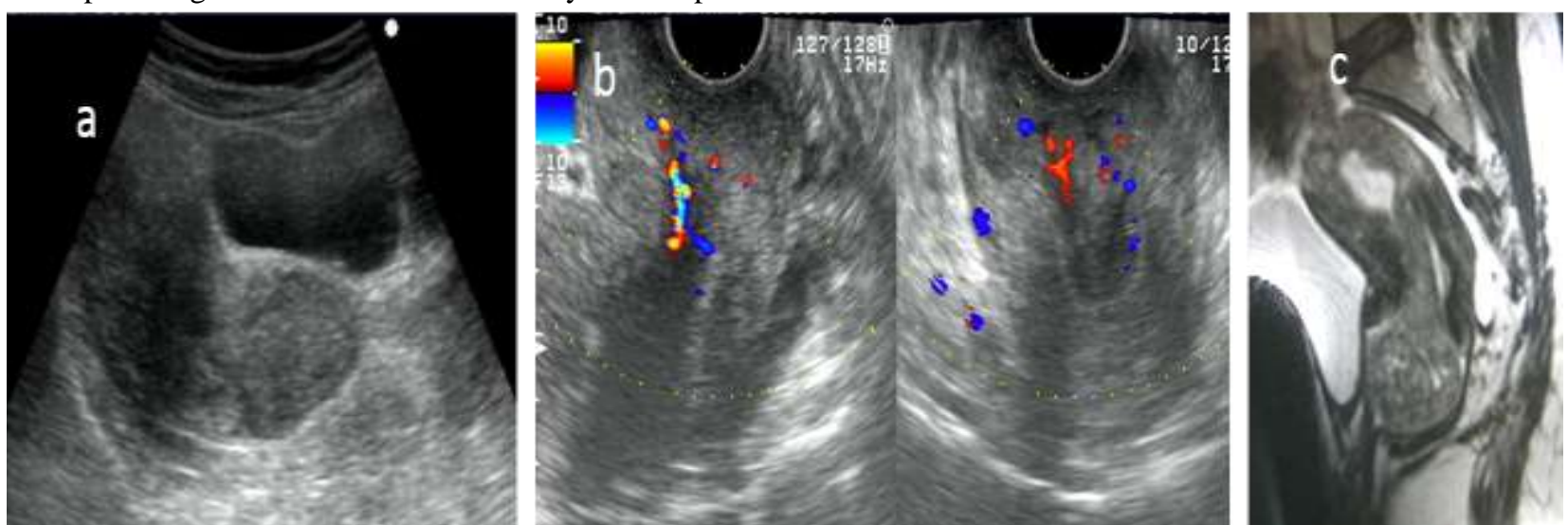

Fig 10a.TAS shows bulky cervix and the margins are not clear. 10b. TVS saggital and coronal Doppler image shows hypoechoic lesion in cervix with pedicle flow suggestive of fibroid polyp, 10c. MRI saggital T2 images show fibroid polyp with long pedicle arising from posterior wall. 
Table1

MEAN AGE OF PATIENT IN EACH FINAL DIAGNOSIS

\begin{tabular}{|l|r|}
\hline FINAL DIAGNOSIS & Mean Age \\
\hline Adenomyosis & 33.67 \\
\hline Fibroid & 39.83 \\
\hline Endometrial Ca & 54.50 \\
\hline Ca cervix & 56.60 \\
\hline Cervical polyp & 35.00 \\
\hline
\end{tabular}

Table 2. Comparison Of Modalities In Myometrial Mass Detection (i) Myometrial Mass Transabdominal, Tv Usg Crosstabulation

\begin{tabular}{|l|l|r|r|r|r|}
\hline \multicolumn{2}{|c|}{} & \multicolumn{3}{|c|}{ TV USG MASS } & \multicolumn{2}{c|}{ Total } \\
\hline \multicolumn{2}{|l|}{} & mass absent & ill defined & present & \\
\hline USG MASS & mass absent & 18 & 2 & 1 & 21 \\
\hline & ill defined & 1 & 2 & 9 & 12 \\
\hline & present & 0 & 0 & 19 & 19 \\
\hline Total & 19 & 4 & 29 & 52 \\
\hline
\end{tabular}

Diagonal Agreement ;75\%

Cohen`S Kappa $(\mathrm{K}) \quad ; 0.6, \mathrm{Z} \quad ; 6.16, \mathrm{P} \leq 0.01$

Myometrial Mass Transabdominal Usg, Mri Crosstabulation

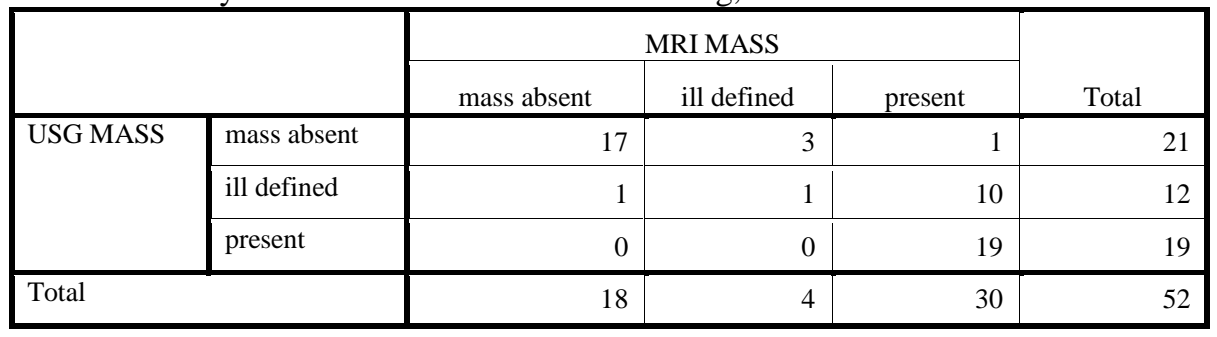

Diagonal Agreement ; 71\%,

Cohen`S Kappa $\quad(\mathrm{K}) \quad ; 0.54, \quad \mathrm{Z} \quad ; 5.6, \quad \mathrm{P} \quad \leq 0.01$

Myomerial Mass Transvaginal Usg, Mri Crosstabulation

\begin{tabular}{|c|c|c|c|c|c|}
\hline & & \multicolumn{3}{|c|}{ MRI MASS } & \multirow[t]{2}{*}{ Total } \\
\hline & & mass absent & ill defined & present & \\
\hline \multirow{3}{*}{$\begin{array}{l}\text { TRANS } \\
\text { VAGINAL } \\
\text { USG MASS }\end{array}$} & mass absent & 18 & 1 & 0 & 19 \\
\hline & ill defined & 0 & 3 & 1 & 4 \\
\hline & present & 0 & 0 & 29 & 29 \\
\hline \multicolumn{2}{|l|}{ Total } & 18 & 4 & 30 & 52 \\
\hline
\end{tabular}

Diagonal Agreement ; 96\%

Cohen`S Kappa $(\mathrm{K}) \quad ; 0.93, \quad \mathrm{Z} \quad ; 8.09, \quad \mathrm{P} \leq 0.01$ 
Table 3 Categorising Myometrial Mass Transabdominal Usg, Mri Crosstabulation

\begin{tabular}{|c|c|c|c|c|c|c|c|}
\hline & \multicolumn{5}{|c|}{ MRI } & \multirow{3}{*}{$\begin{array}{r}\text { Total } \\
\\
0\end{array}$} \\
\hline & & \multirow{2}{*}{$\begin{array}{r}\text { submucosal } \\
0 \\
\end{array}$} & \multirow{2}{*}{$\begin{array}{r}\text { intramural } \\
0 \\
\end{array}$} & \multirow{2}{*}{$\begin{array}{r}\text { subsero } \\
0 \\
\end{array}$} & \multirow{2}{*}{$\begin{array}{r}\text { subsero+ } \\
\text { intramur } \\
0 \\
\end{array}$} & \multirow{2}{*}{$\begin{array}{r}\text { submuco+intramur+subsero } \\
0 \\
\end{array}$} & \\
\hline USG & submuco & & & & & & \\
\hline & intramural & 5 & 6 & 0 & 0 & $\begin{array}{ll} & 0 \\
\end{array}$ & 11 \\
\hline & subsero & 0 & 1 & 1 & 0 & 0 & 2 \\
\hline & $\begin{array}{l}\text { subsero+ } \\
\text { intramur }\end{array}$ & 0 & 0 & 0 & 6 & 5 & 11 \\
\hline \multicolumn{2}{|l|}{ Total } & 5 & 7 & 1 & 6 & 5 & 24 \\
\hline
\end{tabular}

Diagonal Agreement ; $54 \%$

Cohen`S Kappa $(\mathrm{K}) \quad ; 0.38, \quad \mathrm{Z} \quad ; 3.89, \quad \mathrm{P} \leq 0.01$

Categorising Myometrial Mass Transvaginal Usg, Mri Crosstabulation

\begin{tabular}{|c|c|c|c|c|c|c|c|}
\hline & \multicolumn{5}{|c|}{ MRI } & \multirow[t]{2}{*}{ Total } \\
\hline & & submucosal & intramural & subsero & $\begin{array}{l}\text { subsero+ } \\
\text { intramur }\end{array}$ & submuco+intramur+subsero & \\
\hline \multirow{4}{*}{$\begin{array}{l}\text { TV } \\
\text { USG }\end{array}$} & submuco & 2 & 0 & 0 & 0 & 0 & 2 \\
\hline & intramu & 3 & 7 & 0 & 0 & 0 & 10 \\
\hline & subsero & 0 & 0 & 1 & 0 & 0 & 1 \\
\hline & $\begin{array}{l}\text { subsero+ } \\
\text { intramur }\end{array}$ & 0 & 0 & 0 & 6 & 5 & 11 \\
\hline \multicolumn{2}{|l|}{ Total } & 5 & 7 & 1 & 6 & 5 & 24 \\
\hline
\end{tabular}

Diagonal Agreement ; 67\%,

Cohen 'S Kappa $(K) \quad ; 0.55, Z \quad ; 5.24, \quad P \leq 0.01$

\section{Conclusion}

Comparison of modalities to detect myometrial masses, USG is the best screening modality. Calcific degeneration is better identified by TAS and TVS.Cystic degeneration is better identified with TVS and MRI. Submucosal lesions are better characterised by MRI than TVS. Localizing the site of lesion and number of lesions is best with MRI. Exact measurements, circumference of the lesion and degenerative changes can be told by MRI. MRI is the modality of choice to differentiate fibroid from adenomyosis. All the lesions of the uterus are better characterised by MRI. As the surgical approach for fibroid and adenomyosis are drastically different, MRI should be used in preoperative evaluation of all suspected cases of adenomyosis and infertility.

All infertile women going for uterine preservation surgery should undergo MRI before planning the surgical procedure. The exact extent of the mass lesions was better depicted in MRI which helps in staging the tumour.Finally we conclude that pelvic MR Imaging compared to TAS and TVS is a well tolerated, non invasive and accurate modality to characterise uterine mass lesions with excellent histopathological correlation. It is therefore an ideal and accurate preoperative imaging modality for characterising, localizing and detecting number \& extent of uterine mass lesions and for staging

\section{References}

[1]. Padubidri VG, Daftary SN. Shaw's Textbook of gynecology. Elsevier Health Sciences; 2014 Dec 11.

[2]. Murase E, Siegelman ES, Outwater EK, Perez-Jaffe LA, Tureck RW. Uterine Leiomyomas: Histopathologic Features, MR Imaging Findings, Differential Diagnosis, and Treatment 1. Radiographics. 1999 Sep;19(5):1179-97.

[3]. Dudiak CM, Turner DA, Patel SK, Archie JT, Silver B, Norusis M. Uterine leiomyomas in the infertile patient: preoperative localization with MR imaging versus US and hysterosalpingography. Radiology. 1988 Jun;167(3):627-30.

[4]. Zawin M, McCarthy S, Scoutt LM, Comite F. High-field MRI and US evaluation of the pelvis in women with leiomyomas. Magnetic resonance imaging. 1990 Jan 1;8(4):371-6.

[5]. Kang S, Turner DA, Foster GS, Rapoport MI, Spencer SA, Wang JZ. Adenomyosis: specificity of 5 mm as the maximum normal uterine junctional zone thickness in MR images. AJR. American journal of roentgenology. 1996 May;166(5):1145-50.

[6]. Ascher SM, Arnold LL, Patt RH, Schruefer JJ, Bagley AS, Semelka RC, Zeman RK, Simon JA. Adenomyosis: prospective comparison of MR imaging and transvaginal sonography. Radiology. 1994 Mar;190(3):803-6.

[7]. Tamai K, Togashi K, Ito T, Morisawa N, Fujiwara T, Koyama T. MR Imaging Findings of Adenomyosis: Correlation with Histopathologic Features and Diagnostic Pitfalls 1. Radiographics. 2005 Jan;25(1):21-40.

[8]. Mark AS, Hricak H, Heinrichs LW, Hendrickson MR, Winkler ML, Bachica JA, Stickler JE. Adenomyosis and leiomyoma: differential diagnosis with MR imaging. Radiology. 1987 May;163(2):527-9.

[9]. Reinhold C, Atri M, Mehio A, Zakarian R, Aldis AE, Bret PM. Diffuse uterine adenomyosis: morphologic criteria and diagnostic accuracy of endovaginal sonography. Radiology. 1995 Dec;197(3):609-14.

[10]. Byun JY, Kim SE, Choi BG, Ko GY, Jung SE, Choi KH. Diffuse and Focal Adenomyosis: MR Imaging Findings 1. Radiographics. 1999 Oct;19(suppl_1):S161-70.

[11]. Sugimura K, Okizuka H, Imaoka I, Kaji Y, Takahashi K, Kitao M, Ishida T. Pelvic endometriosis: detection and diagnosis with chemical shift MR imaging. Radiology. 1993 Aug;188(2):435-8. 
[12]. Büyük E, Durmuşoĝlu F, Erenus M, Karakoç B. Endometrial disease diagnosed by transvaginal ultrasound and dilatation and curettage. Acta obstetricia et gynecologica Scandinavica. 1999 Jan 1;78(5):419-22.

[13]. Yamashita Y, Mizutani H, Torashima M, Takahashi M, Miyazaki K, Okamura H, Ushijima H, Ohtake H, Tokunaga T. Assessment of myometrial invasion by endometrial carcinoma: transvaginal sonography vs contrast-enhanced MR imaging. AJR. American journal of roentgenology. $1993 \mathrm{Sep} ; 161(3): 595-9$.

[14]. Ascher SM, Scoutt LM, McCarthy SM, Lange RC, DeCherney AH. Uterine changes after dilation and curettage: MR imaging findings. Radiology. 1991 Aug;180(2):433-5.

[15]. Yamashita Y, Harada M, Sawada T, Takahashi M, Miyazaki K, Okamura H. Normal uterus and FIGO stage I endometrial carcinoma: dynamic gadolinium-enhanced MR imaging. Radiology. 1993 Feb;186(2):495-501.

[16]. Shiraiwa M, Joja I, Asakawa T, Okuno K, Shibutani O, Akamatsu N, Kudo T, Hiraki Y. Cervical carcinoma: efficacy of thinsection oblique axial T2-weighted images for evaluating parametrial invasion. Abdominal imaging. 1999 Sep 18;24(5):514-9.

[17]. Outwater EK, Siegelman ES, Chiowanich P, Kilger AM, Dunton CJ, Talerman A. Dilated fallopian tubes: MR imaging characteristics. Radiology. 1998 Aug;208(2):463-9.

[18]. Panageas E, Kier R, McCauley TR, McCarthy S. Submucosal uterine leiomyomas: diagnosis of prolapse into the cervix and vagina based on MR imaging. AJR. American journal of roentgenology. 1992 Sep;159(3):555-8. 